\title{
Guideline update for the performance of fusion procedures for degenerative disease of the lumbar spine. Part 3: Assessment of economic outcome
}

\author{
Zoher Ghogawala, M.D., ${ }^{1}$ Robert G. Whitmore, M.D., ${ }^{1}$ William C. Watters III, M.D., ${ }^{2}$ \\ Alok Sharan, M.D., ${ }^{3}$ Praveen V. Mummaneni, M.D. ${ }^{4}$ Andrew T. Dailey, M.D., 5 \\ Tanvir F. Choudhri, M.D. ${ }^{6}$ Jason C. Eck, D.O., M.S., ${ }^{7}$ Michael W. Groff, M.D., ${ }^{8}$ \\ Jeffrey C. Wang, M.D.,${ }^{9}$ Daniel K. Resnick, M.D. ${ }^{10}{ }^{\text {SANJay S. Dhall, M.D. }}{ }^{4}$ AND \\ Michael G. Kaiser, M.D. ${ }^{11}$
}

${ }^{1}$ Alan and Jacqueline Stuart Spine Research Center, Department of Neurosurgery, Lahey Clinic, Burlington, and Tufts University School of Medicine, Boston, Massachusetts; ${ }^{2}$ Bone and Joint Clinic of Houston, Houston, Texas; ${ }^{3}$ Department of Orthopaedic Surgery, Montefiore Medical Center, Albert Einstein College of Medicine, Bronx, New York; ${ }^{4}$ Department of Neurological Surgery, University of California, San Francisco, California; ${ }^{5}$ Department of Neurosurgery, University of Utah, Salt Lake City, Utah; ${ }^{6}$ Department of Neurosurgery, Icahn School of Medicine at Mount Sinai, New York, New York; ${ }^{7}$ Center for Sports Medicine and Orthopaedics, Chattanooga, Tennessee; ${ }^{8}$ Department of Neurosurgery, Brigham and Women's Hospital, Boston, Massachusetts; ${ }^{9}$ Department of Orthopaedic Surgery, Keck School of Medicine, University of Southern California, Los Angeles, California; ${ }^{10}$ Department of Neurosurgery, University of Wisconsin, Madison, Wisconsin; and ${ }^{11}$ Department of Neurosurgery, Columbia University, New York, New York

A comprehensive economic analysis generally involves the calculation of indirect and direct health costs from a societal perspective as opposed to simply reporting costs from a hospital or payer perspective. Hospital charges for a surgical procedure must be converted to cost data when performing a cost-effectiveness analysis. Once cost data has been calculated, quality-adjusted life year data from a surgical treatment are calculated by using a preferencebased health-related quality-of-life instrument such as the EQ-5D. A recent cost-utility analysis from a single study has demonstrated the long-term (over an 8-year time period) benefits of circumferential fusions over stand-alone posterolateral fusions. In addition, economic analysis from a single study has found that lumbar fusion for selected patients with low-back pain can be recommended from an economic perspective. Recent economic analysis, from a single study, finds that femoral ring allograft might be more cost-effective compared with a specific titanium cage when performing an anterior lumbar interbody fusion plus posterolateral fusion.

(http://thejns.org/doi/abs/10.3171/2014.4 SPINE14259)

KEY WORDS $\quad$ fusion $\quad \bullet \quad$ lumbar spine $\quad$ cost-effectiveness $\bullet \quad$ outcomes
practice guidelines

\section{Recommendations}

There is no evidence that conflicts with the previous recommendations published in the original version of the

Abbreviations used in this paper: ALIF = anterior lumbar interbody fusion; CCR = cost-to-charge ratio; CMS = Center for Medicare and Medicaid Services; CPT $=$ Current Procedural Terminology; DRG = Diagnosis-Related Group; FRA = femoral ring allograft; $\mathrm{HR}-\mathrm{QOL}=$ health-related quality of life; ICBG = iliac crest bone graft; ICD = International Classification of Diseases; ICER = incremental cost-effectiveness ratio; LOS = length of hospital stay; MIS = minimally invasive surgery; ODI = Oswestry Disability Index; $\mathrm{QALY}=$ quality-adjusted life year; rhBMP-2 = recombinant human bone morphogenetic protein-2; SEK = Swedish kronor; SF-36 = 36-Item Short Form Health Survey; SPORT = Spine Patient Outcomes Research Trial; TC = titanium cage; TDR = total lumbar disc replacement; TLIF = transforaminal lumbar interbody fusion; $\mathrm{UK}=$ United Kingdom; VAS = visual analog scale.
"Guidelines for the performance of fusion procedures for degenerative disease of the lumbar spine."

\section{Grade B}

There is Level I evidence (single study) to recommend the use of a circumferential fusion (ALIF + posterolateral fusion) as a more cost-effective option (over an 8-year time period) than stand-alone posterolateral fusion.

There is Level I evidence (single study) to recommend either total lumbar disc replacement (TDR) or lumbar fusion from an economic perspective for the treatment of selected patients with chronic low back pain (over a 2-year time period).

\section{Grade C}

With respect to the combination of anterior lumbar interbody fusion (ALIF) plus posterolateral fusion, there 
is Level II evidence that the use of a femoral ring allograft for interbody fusion is a more cost-effective interbody option than the use of a specific titanium cage.

From an economic perspective, both iliac crest bone graft and recombinant human bone morphogenetic protein-2 (rhBMP-2) are posterolateral fusion graft options in patients over the age of 60 (Level IV evidence).

From an economic perspective, both minimally invasive and open transforaminal lumbar interbody fusion (TLIF) techniques are options when treating patients with symptomatic Grade I degenerative spondylolisthesis (Level IV evidence).

\section{Grade I}

There are conflicting data regarding the cost-effectiveness of cell-salvage auto-transfusion as an adjunct to lumbar fusion (Level IV evidence).

\section{Rationale}

Ongoing changes in national health care policy have created an increased awareness on medical resource allocation and greater emphasis on cost-benefit analyses. As part of the American Recovery and Reinvestment Act of 2009, the federal government has allocated \$1.1 billion in funds toward comparative-effectiveness research., ${ }^{9,23}$ An area of specific interest has been the application of lumbar fusion in the management of degenerative spine disease, with a focus on establishing clinical efficacy and cost-effectiveness. ${ }^{31}$ Management of chronic degenerative spinal conditions in the United States is estimated to cost nearly $\$ 85$ billion annually, with a significant percentage attributed to the dramatic increase in the frequency of lumbar fusion procedures..$^{13,15,25}$ In 2004, more than 300,000 spinal fusions were performed in the US, accounting for more than $\$ 16$ billion in hospital charges alone. ${ }^{14}$ Advances in surgical fusion technologies have improved the surgeon's ability to attain a solid arthrodesis and expand the treatment options available for patients with spine disorders. Since the initial publication of the Lumbar Fusion Guidelines, there is recognition that the evolution of devices and techniques for lumbar fusion impacts not only surgical outcomes but also health care costs. The purpose of this qualitative review is to evaluate current research that examines the economic impact of lumbar fusion on the management of degenerative lumbar spine disease. The expense of fusion surgery and new fusion technologies must be weighed against the incremental improvement in patient outcomes and quality of life.

\section{Search Criteria}

A search of the National Library of Medicine database of literature was performed with limits: (("2002" [PDAT]: “2011”[PDAT]) AND English[lang]). Using the following terms: ("lumbar" AND "fusion"[All Fields]) yielded 4002 citations. The following terms were combined: ("lumbar" AND "fusion" AND "outcomes"[All Fields]), which yielded 807 citations and ("lumbar" AND "fusion" AND "cost"[All Fields]) which yielded 154 cita- tions. The titles and abstracts of the 154 articles were reviewed. In addition, additional searches were performed with terms: (("lumbar fusion"'[MeSH]) AND ("cost effectiveness"[MeSH]) OR ("employment status"[MeSH]), ("mortality") OR ("medical care costs[MeSH])" OR ("cost containment" [MeSH] OR “cost comparison"'[MeSH]) OR ("spondylolisthesis")). Of the articles reviewed, 13 clinical series focusing on adult patients who underwent lumbar fusion procedures were selected based on the inclusion of an economic analysis.

A comprehensive economic analysis from a societal perspective that included multivariate sensitivity analyses was performed in 4 articles. ${ }^{16,18,36,39}$ A cost analysis investigating various surgical approaches for lumbar fusion was performed in 1 study. ${ }^{36}$ Another study performed a cost analysis on the type of interbody device used in lumbar fusion. ${ }^{16}$ Two randomized trials compared outcomes and cost of lumbar fusion to conservative management. ${ }^{19,39}$ Two studies examined the comparative costeffectiveness of minimally invasive versus open TLIF. ${ }^{43,44}$ One preliminary study provided cost-effectiveness data for TLIF procedures. ${ }^{1}$ Six studies addressed incremental cost-effectiveness of new technology for lumbar fusion. . $^{2,7,20,30,34}$

\section{Scientific Foundation}

A cost-utility analysis is a specific type of cost-effectiveness evaluation that allows a comparison of 2 alternative treatment strategies in terms of the cost required for a given clinical outcome. These analyses are measured in terms of quality-adjusted life years (QALYs) gained, taking into account both the quantity and quality of life resulting from a given intervention. ${ }^{21,32}$ For calculation of QALYs, patients must be surveyed using a preferencebased health-related quality-of-life (HR-QOL) outcome instrument, such as the EQ-5D (EuroQol Group). ${ }^{29,37}$ Another commonly used preference-based HR-QOL instrument is the SF-6D, ${ }^{4}$ which consists of 11 items selected from the 36-Item Short Form Health Survey (SF-36)..$^{5}$ An HR-QOL score is converted to a "health utility," typically a number on a continuum between 0 , indicating death, and 1 , indicating perfect health. Negative values can be generated when conditions considered worse than death exist. ${ }^{38}$ A QALY is determined by the number of years in a given health state multiplied by the utility score assigned to that particular health state. A single year spent in perfect health is given the value of 1 QALY.

When comparing 2 treatment strategies, $\mathrm{A}$ and $\mathrm{B}$, it is necessary to know the incremental cost-utility ratio (similar to the incremental cost-effectiveness ratio [ICER]). The ICER of Treatment B versus $A$ is calculated as: (Cost of B - Cost of A)/(QALYs gained from B - QALYs gained from $\mathrm{A})$.

From this calculation, the incremental cost of each additional QALY is determined when Treatment B is chosen over Treatment A. The acceptable cost per additional QALY represents society's willingness to pay and serves as a foundation for cost-effectiveness analyses. Since 1982, \$50,000 per QALY gained has been cited as the threshold for a cost-effective intervention, ${ }^{42}$ although 
more recent proposals argue for a cut-off value closer to $\$ 100,000$ or more, reflecting inflation and increased costs for research and development. ${ }^{3}$

There are 3 main categories of cost: direct, indirect, and intangible. Direct costs are resources that are consumed by the surgical procedure (i.e., operating room supplies, surgeon time and labor, cost of hospital stay). Indirect costs generally refer to a loss of productivity due to the morbidity or mortality of the surgical procedure. For example, the amount of work missed by the patient and/or their caretaker during the recovery period would qualify as indirect costs. Intangible costs include the pain and suffering from the surgical procedure. Both the indirect and the intangible costs are often difficult to quantify in monetary terms. Therefore, the total cost of a surgical procedure is based on the quantity of resources used and the assignment of cost to these resources. Determining the quantity of resources used is relatively straightforward for a surgical procedure. However, unit costs may vary between different countries, geographic regions, time periods, or hospitals. The cost perspective ("costs to whom") must be considered and expressly stated in any economic analysis. Costs to the patient for an intervention may be quite different from those to a hospital, a thirdparty payer, or to society itself.

Several methods have been introduced to estimate the total cost of a surgical procedure. One method utilizes the total hospital charge for the procedure and admission. The hospital charge is based on several data coding systems that are currently used to determine reimbursement, including the diagnosis-related group (DRG), International Classification of Diseases (ICD) system, and current procedural terminology (CPT) system. ${ }^{10,12,33,45}$ This method, however, fails to reflect the actual amount of reimbursement received by the hospital or physician, or the actual costs, counting instead upon charges as a surrogate for costs. For the hospital charges of different centers to be used as a proxy for direct costs, a cost-to-charge ratio (CCR) must be calculated and applied. The CCR is specific for every hospital, for many departments within the hospital, and for a given time period. The CCR is calculated from Medicare Hospital Cost Reports (Worksheet C or D) in combination with claims data. Although obtaining CCRs is labor intensive, it has been suggested that this approach is the most accurate way to determine actual "cost" when comparing different centers. ${ }^{17}$ Other methods for calculating cost of a surgical procedure include using total Medicare charges allowed, or the Medicare reimbursement. The most common and simplest way to estimate the direct cost for a procedure is using Medicare payments. ${ }^{35,41}$ Real hospital costs can also be estimated by using the Center for Medicare and Medicaid Services (CMS) reimbursement value for DRG and CPT codes.

Micro-costing methodology involves measuring all the costs and benefits of a treatment as accurately as possible. It becomes particularly useful when evaluating and comparing regional differences in the resource utilization for a particular surgical procedure. However, microcosting analysis is expensive and time-consuming due to extensive record keeping and database management. Of- ten details, specific to a single institution, limit the generalizability of the conclusions. Finally, there are some elements of the micro-costing analysis that inevitably require estimation, which will also compromise the validity of the conclusions. ${ }^{28}$

An understanding of the methodology used to determine cost is critical when interpreting the results and conclusions of a study. ${ }^{11}$ For example, in the recent Spine Patient Outcomes Research Trial (SPORT), the cost-effectiveness of surgery relative to nonoperative treatment for lumbar disc disease was \$69,403/QALY using overall adult surgery costs (all payers), but only $\$ 34,355 / \mathrm{QALY}$ using Medicare population-specific surgery costs. ${ }^{40}$ Using Medicare-based reimbursements will significantly lower the estimate of medical costs for any given treatment, which may be appropriate in older aged individuals, but irrelevant when considering younger patients with better (e.g., Workers Compensation) or worse (e.g., Medicaid) reimbursements.

\section{Literature Review}

\section{Comparison of Lumbar Fusion to Nonoperative Therapy}

Utilizing 2-year follow-up data from the SPORT study, Tosteson et al. investigated the cost-effectiveness of lumbar fusion for patients with degenerative spondylolisthesis and spinal stenosis. ${ }^{39}$ Patients underwent nonoperative treatment, decompressive laminectomy, or laminectomy with fusion, with or without instrumentation and/or iliac crest bone graft. QALYs were calculated from EQ-5D scores at baseline, 6 weeks, and 3, 6, 12, and 24 months following treatment. Direct and indirect costs were collected prospectively based on 2004 Medicare payments. Operative management of spinal stenosis improved health significantly compared with nonoperative care, 0.17 QALYs gained, at a cost of $\$ 77,600$ per QALY. Operative management of degenerative spondylolisthesis, $93 \%$ of which were lumbar fusions, provided significant benefit, with 0.23 QALYs gained at a cost of $\$ 115,600$ per QALY. Although $\$ 115,600$ is greater than the accepted societal expense per QALY, it is much less than previous estimates. Kuntz et al. reported that an instrumented lumbar fusion procedure cost \$3,112,800 per QALY and instead favored noninstrumented fusion with a comparative medical benefit. ${ }^{24}$ Although Tosteson et al. performed a rigorous cost analysis, the underlying heterogeneity of the study population and surgical techniques limits the validity of the study conclusions. In addition, follow-up data beyond 2 years will be essential to order to formulate meaningful recommendations regarding the cost-effectiveness of lumbar fusion over nonfusion treatments for lumbar degenerative disorders. This study provides Level II evidence that surgery for degenerative spondylolisthesis is effective but more costly than surgery for spinal stenosis (see Table 1).

Fritzell et al. performed a randomized controlled trial of patients with chronic low-back pain who underwent either lumbar fusion or nonoperative treatment. ${ }^{19}$ Two hundred eighty-four patients from multiple centers were randomized, and outcomes were measured by the 
Part 3: Assessment of economic outcome

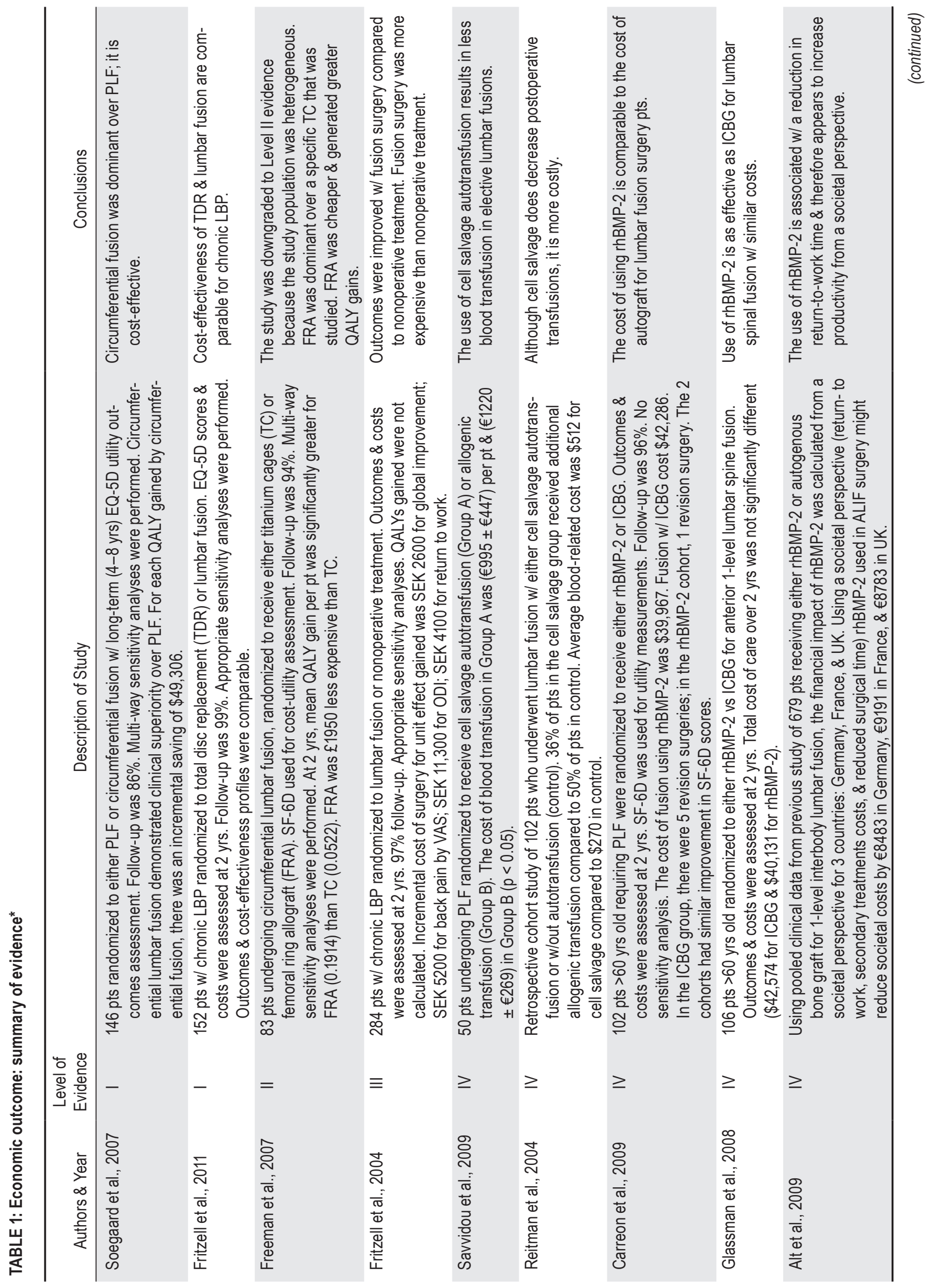




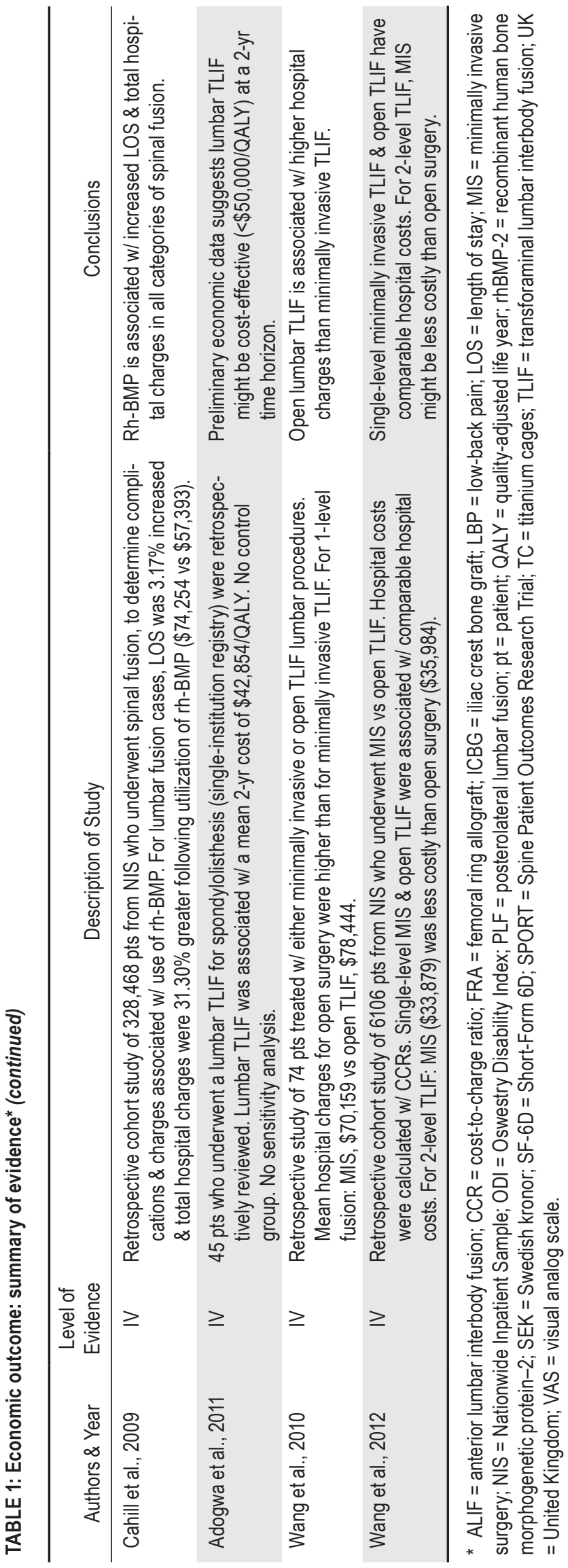

Oswestry Disability Index (ODI) and visual analog scale (VAS) for 2 years. Patients who underwent surgery had 1 of 3 procedures: noninstrumented posterolateral fusion, instrumented posterolateral fusion, or a posterolateral circumferential fusion with pedicle screws and interbody grafts. Both direct and indirect costs were collected for each treatment group. The mean cost per patient was significantly higher in the surgical group (cost in Swedish kronor [SEK]: SEK 123,000 [US\$18,731]) compared with the nonoperative group (SEK 65,200 [US\$9929]). A significantly greater percentage of patients in the surgically treated group returned to part-time or full-time work compared with the nonoperative group (33\% vs $16 \%, \mathrm{p}=$ $0.015)$. Overall, lumbar fusion was associated with significantly greater improvements in pain and function compared with nonoperative treatment at 2 years. However, there was no difference in clinical outcome between the 3 fusion techniques studied. Compared with noninstrumented posterolateral fusion, the placement of pedicle screws increased hospital costs by $66 \%$ and a circumferential fusion procedure increased them by $103 \%$. This study, however, did not measure QALYs, and therefore, it is difficult to interpret and generalize the data against more meaningful cost analyses. ${ }^{26}$ Another significant limitation of this study is that the retrospective nature of the cost analysis, introducing the potential for significant recall bias regarding the patient's recollection of outpatient health resource utilization. Due to these design limitations, no definite conclusions regarding cost-effectiveness can be formulated from this economic analysis. It provides Level III evidence that outcomes are improved with fusion surgery for low-back pain compared with nonoperative treatment (see Table 1).

\section{Lumbar Disc Arthroplasty Versus Posterior Lumbar Fusion}

Fritzell's group performed a randomized controlled trial comparing the cost-effectiveness of total lumbar disc replacement (TDR) versus posterolateral fusion with or without interbody fusion..$^{18}$ One hundred fifty-two patients with chronic low-back pain were randomized to receive either TDR (80 patients) or fusion (72 patients). QALY outcomes were assessed using EQ-5D over a 2-year time period. Direct and indirect health costs were collected. Utilization of all outpatient resources and loss of work productivity were included to calculate health costs from a societal perspective. Multi-way sensitivity analyses were performed on excluding reoperations in both groups, costs for inpatient rehabilitation, and health cost discounting (see Table 1).

Follow-up data were available on $99 \%$ of cases at 2 years. From a societal perspective the mean health cost for TDR was SEK 599,560 (US\$90,162) and for lumbar fusion was SEK 685,919 (US\$103,149). This difference in cost was not statistically significant. At 2 years after surgery both groups demonstrated an improvement of 0.4 QALYs. The study's authors concluded that lumbar TDR and lumbar fusion have similar cost-effectiveness profiles in Sweden, although TDR was associated with lower costs from a health care perspective because of a lower reoperation rate at 2 years (see Table 1). 


\section{Part 3: Assessment of economic outcome}

\section{Circumferential Lumbar Fusion Versus Posterolateral Lumbar Fusion}

Soegaard et al. randomized 146 patients with chronic low-back and leg pain to either posterolateral or circumferentially instrumented fusion and followed outcomes for 4-8 years after surgery. ${ }^{36}$ Outcomes were measured with EQ-5D, SF-36, ODI, and pain scores. Service utilization (i.e., surgery, reoperations, rehospitalizations, general practitioner visits, etc.) was recorded per patient by the National Patient Registry in Denmark. Service utilization is valued by national average unit costs through the DRG system of coding. A micro-costing analysis was performed, including patient costs, medications, and productivity costs. The circumferentially treated group demonstrated better functional outcome $(p=0.004)$, higher fusion rate $(\mathrm{p}<0.04)$, and fewer reoperations $(15 \%$ versus $38 \%$ ) compared with the posterolateral cohort. From a societal perspective, the circumferentially treated group demonstrated significantly lower costs compared with the posterolateral group $(\mathrm{p}=0.012)$, primarily due to the higher reoperation rate and lower return to work rate observed in the posterolateral group. This study showed an incremental savings of $\$ 49,306$ per QALY following a circumferential fusion compared with a posterolateral fusion. The study benefited from the long follow-up interval, large number of patients enrolled, and the comprehensive National Patient Registry. However, there are limitations, which include the heterogeneous population of patients with respect to presenting diagnosis and history of previous spine surgery. For example, patients with isthmic spondylolisthesis may have more favorable outcomes following lumbar fusion than other patients undergoing a lumbar fusion for different reasons. This study provides Level I evidence that circumferential fusion through a posterolateral approach is more cost-effective than stand-alone posterolateral fusion for up to 8 years following surgery (see Table 1).

\section{Minimally Invasive Versus Open TLIF}

Two retrospective studies by Wang et al. have addressed the cost-effectiveness of minimally invasive versus open TLIF. ${ }^{43,44}$ In a retrospective review of 59 singlelevel TLIF cases at one institution using hospital charges as a surrogate for hospitalization costs, Wang and colleagues found that minimally invasive single-level TLIF was associated with lower hospital charges $(\$ 70,159)$ compared with open single-level TLIF $(\$ 78,444)(\mathrm{p}=$ 0.027). Using the Nationwide Inpatient Sample (6106 cases) and applying CCRs to estimate hospital costs, Wang et al. found that minimally invasive single-level fusion was associated with hospital costs similar to those for open TLIF; however, for 2-level procedures, hospital costs of minimally invasive TLIF procedures $(\$ 33,879)$ were lower than costs of open surgery $(\$ 35,984 ; p=0.0023)$. Neither study included outcome measures, so ICERs could not be calculated to assess cost-effectiveness (see Table 1).

A recent preliminary study reported cost-effectiveness data for TLIF using EQ-5D to measure QALYs gained over a 2-year time period. Adogwa et al. calculated health costs from a societal perspective by includ- ing inpatient and outpatient Medicare costs as well as workday losses. ${ }^{1}$ While this type of analysis was limited because there was no comparison group, the results demonstrated that TLIF might be cost-effective $(\$ 42,854 /$ QALY). Comparative studies are needed to calculate ICERs to validate these findings (see Table 1).

\section{Type of Interbody Device Used for Lumbar Interbody Fusion}

Freeman et al. randomized 83 patients with chronic low-back pain undergoing circumferential fusion to receive either a titanium cage (TC) or femoral ring allograft (FRA) in an effort to determine which interbody graft was more cost-effective. ${ }^{16}$ Outcomes were measured by ODI and SF-6D at various intervals for up to 2 years following surgery. Direct cost data were derived from the National Health Service, using local center-specific unit costs for individual health resources. The indirect costs were measured by the human capital approach, which is based on the total expected production losses for an individual worker for the duration of disability. ${ }^{22}$ The cost of TC was approximately 10 times higher than FRA ( $£ 1609.76$ [US\$2583] vs $£ 158.92$ [US\$255]), and the mean total cost per patient was significantly higher in the TC group, $£ 9052$ (US\$14,531) compared with $£ 7102$ (US\$11,399) ( $\mathrm{p}<0.001)$. In addition, the mean QALY gained per patient over 24 months was significantly greater in the FRA group (0.1914) compared with the TC group (0.0522). Because the FRA proved to be less expensive and increased QALYs compared with a TC, an ICER was not necessary. Finally, using the human capital approach to cost productivity, total gross mean earnings in the 2 postoperative years were $£ 7456$ (US\$11,968) in the TC group and $£ 14,517$ (US\$23,303) in the FRA group. A higher percentage of FRA patients had a positive change in employment status following surgery compared with TC patients (21.6\% vs $9.8 \%$ ), and FRA patients were more likely to see an improvement in their salary after surgery. The strength of this study lies in its randomized design, excellent outcomes data, and comprehensive cost analysis. However, some utility data were missing, requiring estimation in the analysis. In addition, a few important health care resources were omitted from the cost analysis, including radiology costs, medication, outpatient services, and primary care costs. The clinical observations of Freeman et al. are supported by an earlier randomized trial by McKenna et al. that found improved clinical outcomes in patients implanted with FRA compared with TC. ${ }^{27}$ These authors speculated that the improved pain relief with FRA may be due to the more physiological transfer of loads as the fusion matures compared with point loading with titanium cages. Overall, the study of Freeman et al. provides Level II evidence that the use of femoral ring allograft for lumbar interbody fusion is more cost-effective than the use of a specific titanium cage when performing a posterolateral circumferential fusion (see Table 1).

\section{Cost-Effectiveness of rhBMP}

Cahill et al. reviewed a retrospective cohort of 328,468 patients undergoing lumbar fusion procedures 


\section{Z. Ghogawala et al.}

from 2002 to 2006, selected from the Nationwide Inpatient Sample database, in an effort to determine the cost associated with the application of recombinant human bone morphogenetic protein (rhBMP) as a fusion enhancer. ${ }^{7}$ The usage of rhBMP has increased from $0.69 \%$ of all fusions in 2002 to $24.89 \%$ of all fusions in 2006 . The primary outcome measures included the rate of rhBMP utilization, complications, length of stay, and associated hospital charges. There were no differences in the rates of overall complications based on the application of rhBMP. The use of rhBMP was associated with an extended hospitalization ( $3.17 \%$ increase). The utilization of rhBMP was associated with a $20 \%$ increase in total hospital charges compared with those undergoing fusion without rhBMP (\$74,254 vs \$57,393). This analysis, however, is limited by the lack of clinical outcome data, and since only total hospital charges were considered, with a wide range in charges identified between different institutions, \$54,737-\$102,663 for lumbar fusions supplemented with rhBMP and \$39,660-\$83,608 when rhBMP was not included. This study provides Level IV evidence that rhBMP is associated with increased length of hospital stay (LOS) and total hospital charges in all categories of spinal fusion (see Table 1).

Glassman et al. conducted a randomized trial of rhBMP-2 versus iliac crest bone graft (ICBG) in patients over 60 years of age undergoing either single-level or multilevel instrumented posterolateral lumbar fusion..$^{20}$ Outcomes were measured up to 2 years after surgery utilizing validated outcomes instruments, including the ODI, SF-36, and numerical pain scales. Direct costs were obtained from actual reimbursement to the hospital. Where actual payments could not be determined, a Medicare fee schedule was used to assign direct cost. No significant differences in any of the outcome measures were identified between the 2 treatment groups. However, the fusion rate at 2 years, as measured by CT and presence of bridging bone, was significantly higher in the rhBMP-2 group $(86.3 \%$ vs $70.8 \%, p=0.030)$. Including revision surgery and outpatient costs (i.e., postoperative rehabilitation), the total cost of care over 2 years was not significantly different for 2 groups $(\$ 42,574$ for the ICBG group vs $\$ 40,131$ for the rhBMP-2 group). This study provides Level IV evidence that either ICBG or rhBMP-2 may be considered as posterolateral fusion graft options in patients over the age of 60 (see Table 1).

Using the same data as Glassman et al., Carreon et al. performed a cost-utility analysis of rhBMP-2 versus ICBG. ${ }^{8}$ Utility was estimated from the SF-6D. ${ }^{4}$ As described above, costs were determined from actual reimbursement to hospitals and physicians participating in the trial and included reimbursement for inpatient and outpatient services, radiographic imaging, and medications. There was no significant difference in change in utility between the 2 groups at any time point. The total cost of using rhBMP-2 was $\$ 39,967$, with a 0.11 mean improvement in SF-6D; for ICBG, the cost was \$42,286, with a mean improvement of 0.10 in SF-6D. The authors failed to account for indirect costs such as lost wages or out-ofpocket expenses. This study provides Level IV evidence that the cost of using rhBMP-2 is comparable to the cost of autograft for patients undergoing lumbar fusion surgery (see Table 1).

Alt et al. performed a cost analysis on a previously studied cohort of patients who either received rhBMP-2 or ICBG during lumbar fusion., ${ }^{2,6}$ The study population included 279 patients randomized to either rhBMP-2 or ICBG and 400 patients from a prospective nonrandomized cohort. The original trial demonstrated significant reduction in surgery time (reduction of 54 minutes) and blood loss (reduction of $66 \mathrm{ml}$ ) when rhBMP-2 was used instead of ICBG. Patients in the rhBMP-2 group required fewer revision operations and returned to work earlier. Finally, the fusion rate and clinical outcomes of patients in the rhBMP-2 group were significantly better than those in the ICBG group. The authors performed a retrospective cost analysis from data collected in 3 countries: Germany, France, and the United Kingdom (UK). Direct costs were estimated from the expense associated with the use of rhBMP-2, operating room time, and revision surgery. Indirect costs were estimated from the loss of productivity and the national average of gross wages. From a societal perspective, the overall savings associated with use of rhBMP-2 compared with ICBG for Germany, France, and the UK were $€ 8483$ (US\$11,745), $€ 9191$ (US\$12,726), and $€ 8783$ (US\$12,161), respectively. This study is limited by the retrospective study design and failure to account for costs associated with out-of-pocket expenses, medications, or outpatient treatment. This study provides Level IV evidence that the use of rhBMP-2 is associated with a reduction in return-to-work time and may increase productivity from a societal perspective.

\section{Conflicting Data Regarding the Cost-Effectiveness of Cell-Salvage Autotransfusion}

Savvidou et al. randomized 50 patients undergoing instrumented lumbar fusion to a group that received cellsalvage autotransfusion and a group that did not. ${ }^{34}$ The total amount of allogeneic and cell-salvage blood used per patient was recorded. The cost for each unit of allogeneic blood ( $€ 450$ [US\$623]) versus cell-salvage blood $(€ 370$ [US\$512]) was determined from the Greek ministry of health. The total transfusion cost in the group that received cell-salvage autotransfusion was significantly lower than the group that did not (€995 [US\$1377] versus $€ 1220$ [US\$1689], $\mathrm{p}<0.05)$. Because this study did not measure outcomes, it is impossible to perform an adequate cost-effectiveness analysis regarding the use of autotransfusion. This study provides Level IV evidence that the use of cell-salvage autotransfusion lowered the costs of transfusing blood for elective lumbar fusions (see Table 1).

Reitman et al. performed a retrospective analysis of patients who had undergone instrumented lumbar fusion with and without cell-salvage autotransfusion. ${ }^{30}$ There was no significant difference in blood loss between the 2 groups, and $38 \%$ of the blood in the study group was returned as cell-salvage autotransfusion. The average charge of the cell-salvage was $\$ 512$ per patient compared with $\$ 270$ per patient in the control group. The authors concluded that the cost of blood replacement was higher in the cell-salvage autotransfusion group. As with other studies investigating the application of cell-salvage autotransfu- 
sion, there are insufficient data to perform a meaningful cost-effectiveness analysis. This study provides Level IV evidence that use of cell-salvage autotransfusion is more costly than normal postoperative transfusion (see Table 1).

\section{Summary}

Lumbar fusion for certain degenerative spine disorders can be effective in improving clinical outcomes and long-term quality of life when compared with nonoperative therapy. Comprehensive economic analyses that include long-term clinical outcomes data and both direct and indirect costs will be necessary before any recommendations can be made regarding the cost-effectiveness of various methods of lumbar fusion. Given the significant impact of lumbar degenerative disease, it is essential from a societal perspective that these studies be conducted. Recent costanalyses have demonstrated the long-term benefits of circumferential fusions over posterolateral fusions, FRA over TC when performing an interbody fusion, and that both rhBMP-2 and ICBG are associated with similar costs.

\section{Key Issues for Future Investigation}

As new technologies for lumbar fusion are introduced and studies are performed to assess their effectiveness, the inclusion of an economic analysis is essential. Appropriate long-term follow-up is important when designing trials, as the benefits of lumbar fusion, both clinically and economically, may be apparent several years following the operation. The major challenge for investigators is to determine the most reliable estimate of cost. Medicare reimbursement may underestimate real costs, and medical charges may grossly overestimate true costs. New methodology is needed to allow for meaningful long-term assessment of health cost, and it may be that the best "laboratory" for these studies is a well-run health care system that follows costs directly along with patient outcomes as a matter of course.

\section{Acknowledgments}

We would like to acknowledge the AANS/CNS Joint Guidelines Committee (JGC) for their review, comments, and suggestions; Laura Mitchell, CNS Guidelines Project Manager, for her organizational assistance; and Linda O'Dwyer, medical librarian, for assistance with the literature searches. We would also like to acknowledge the following individual JGC members for their contributions throughout the review process: Timothy Ryken, M.D.; Kevin Cockroft, M.D.; Sepideh Amin-Hanjani, M.D.; Steven N. Kalkanis, M.D.; John O'Toole, M.D., M.S.; Steven Casha, M.D., Ph.D.; Aaron Filler, M.D., Ph.D., F.R.C.S.; Daniel Hoh, M.D.; Steven Hwang, M.D.; Todd McCall, M.D.; Jeffrey J. Olson, M.D.; Julie Pilitsis, M.D., Ph.D.; Joshua Rosenow, M.D.; and Christopher Winfree, M.D.

\section{Disclosure}

Administrative costs of this project were funded by the Congress of Neurological Surgeons and the Joint Section on Disorders of the Spine and Peripheral Nerves of the American Association of Neurological Surgeons and Congress of Neurological Surgeons. No author received payment or honorarium for time devoted to this project. Dr. Ghogawala receives grants from the Patient
Centered Outcomes Research Institute (PCORI) and the National Institutes of Health (NIH). Dr. Groff is a consultant for DePuy Spine and EBI Spine. Dr. Mummaneni owns stock in Spinicity and receives honoraria from DePuy Spine and Globus and royalties from DePuy Spine, Quality Medical Publishers, and Thieme Publishing. Dr. Wang owns stock in Bone Biologics, AxioMed, Amedica, CoreSpine, Expanding Orthopedics, Pioneer, Syndicom, VG Innovations, PearlDiver, Flexuspine, Axis, FzioMed, Benvenue, Promethean, Nexgen, ElectroCore, and Surgitech and holds patents with and receives royalties from Biomet, Stryker, SeaSpine, Aesculap, Osprey, Amedica, Synthes, and Alphatec. The authors report no other potential conflicts of interest concerning the materials or methods used in this study or the findings specified in this paper.

Author contributions to the study and manuscript preparation include the following. Acquisition of data: all authors. Analysis and interpretation of data: all authors. Drafting the article: Ghogawala. Critically revising the article: all authors. Reviewed submitted version of manuscript: all authors. Approved the final version of the manuscript on behalf of all authors: Ghogawala. Study supervision: Kaiser.

\section{References}

1. Adogwa O, Parker SL, Davis BJ, Aaronson O, Devin C, Cheng JS, et al: Cost-effectiveness of transforaminal lumbar interbody fusion for Grade I degenerative spondylolisthesis. Clinical article. J Neurosurg Spine 15:138-143, 2011

2. Alt V, Chhabra A, Franke J, Cuche M, Schnettler R, Le Huec JC: An economic analysis of using rhBMP-2 for lumbar fusion in Germany, France and UK from a societal perspective. Eur Spine J 18:800-806, 2009

3. Braithwaite RS, Meltzer DO, King JT Jr, Leslie D, Roberts MS: What does the value of modern medicine say about the $\$ 50,000$ per quality-adjusted life-year decision rule? Med Care 46:349-356, 2008

4. Brazier J, Roberts J, Deverill M: The estimation of a preference-based measure of health from the SF-36. J Health Econ 21:271-292, 2002

5. Brazier J, Usherwood T, Harper R, Thomas K: Deriving a preference-based single index from the UK SF-36 Health Survey. J Clin Epidemiol 51:1115-1128, 1998

6. Burkus JK, Heim SE, Gornet MF, Zdeblick TA: Is INFUSE bone graft superior to autograft bone? An integrated analysis of clinical trials using the LT-CAGE lumbar tapered fusion device. J Spinal Disord Tech 16:113-122, 2003

7. Cahill KS, Chi JH, Day A, Claus EB: Prevalence, complications, and hospital charges associated with use of bonemorphogenetic proteins in spinal fusion procedures. JAMA 302:58-66, 2009

8. Carreon LY, Glassman SD, Djurasovic M, Campbell MJ, Puno RM, Johnson JR, et al: RhBMP-2 versus iliac crest bone graft for lumbar spine fusion in patients over 60 years of age: a costutility study. Spine (Phila Pa 1976) 34:238-243, 2009

9. Chalkidou K, Whicher D, Kary W, Tunis S: Comparative effectiveness research priorities: identifying critical gaps in evidence for clinical and health policy decision making. Int J Technol Assess Health Care 25:241-248, 2009

10. Chulis GS: Assessing Medicare's prospective payment system for hospitals. Med Care Rev 48:167-206, 1991

11. Chumney EC, Biddle AK, Simpson KN, Weinberger M, Magruder KM, Zelman WN: The effect of cost construction based on either DRG or ICD-9 codes or risk group stratification on the resulting cost-effectiveness ratios. Pharmacoeconomics 22:1209-1216, 2004

12. Chute CG, Cohn SP, Campbell KE, Oliver DE, Campbell JR: The content coverage of clinical classifications. J Am Med Inform Assoc 3:224-233, 1996

13. Davis H: Increasing rates of cervical and lumbar spine sur- 
gery in the United States, 1979-1990. Spine (Phila Pa 1976) 19:1117-1124, 1994

14. Deyo RA: Back surgery-who needs it? N Engl J Med 356:2239-2243, 2007

15. Deyo RA, Cherkin D, Conrad D, Volinn E: Cost, controversy, crisis: low back pain and the health of the public. Annu Rev Public Health 12:141-156, 1991

16. Freeman BJ, Steele NA, Sach TH, Hegarty J, Soegaard R: ISSLS prize winner: cost-effectiveness of two forms of circumferential lumbar fusion: a prospective randomized controlled trial. Spine (Phila Pa 1976) 32:2891-2897, 2007

17. Friedman B, De La Mare J, Andrews R, McKenzie DH: Practical options for estimating cost of hospital inpatient stays. J Health Care Finance 29:1-13, 2002

18. Fritzell P, Berg S, Borgström F, Tullberg T, Tropp H: Cost effectiveness of disc prosthesis versus lumbar fusion in patients with chronic low back pain: randomized controlled trial with 2-year follow-up. Eur Spine J 20:1001-1011, 2011

19. Fritzell P, Hägg O, Jonsson D, Nordwall A, Swedish Lumbar Spine Study Group: Cost-effectiveness of lumbar fusion and nonsurgical treatment for chronic low back pain in the Swedish Lumbar Spine Study: a multicenter, randomized, controlled trial from the Swedish Lumbar Spine Study Group. Spine (Phila Pa 1976) 29:421-434, 2004

20. Glassman SD, Carreon LY, Djurasovic M, Campbell MJ, Puno RM, Johnson JR, et al: RhBMP-2 versus iliac crest bone graft for lumbar spine fusion: a randomized, controlled trial in patients over sixty years of age. Spine (Phila Pa 1976) 33:28432849, 2008

21. Gold MR, Franks P, McCoy KI, Fryback DG: Toward consistency in cost-utility analyses: using national measures to create condition-specific values. Med Care 36:778-792, 1998

22. Korthals-de Bos I, van Tulder M, van Dieten H, Bouter L: Economic evaluations and randomized trials in spinal disorders: principles and methods. Spine (Phila Pa 1976) 29:442448, 2004

23. Kuehn BM: Institute of Medicine outlines priorities for comparative effectiveness research. JAMA 302:936-937, 2009

24. Kuntz KM, Snider RK, Weinstein JN, Pope MH, Katz JN: Cost-effectiveness of fusion with and without instrumentation for patients with degenerative spondylolisthesis and spinal stenosis. Spine (Phila Pa 1976) 25:1132-1139, 2000

25. Martin BI, Deyo RA, Mirza SK, Turner JA, Comstock BA, Hollingworth W, et al: Expenditures and health status among adults with back and neck problems. JAMA 299:656-664, 2008

26. McCabe C, Claxton K, Culyer AJ: The NICE cost-effectiveness threshold: what it is and what that means. Pharmacoeconomics 26:733-744, 2008

27. McKenna PJ, Freeman BJ, Mulholland RC, Grevitt MP, Webb JK, Mehdian SH: A prospective, randomised controlled trial of femoral ring allograft versus a titanium cage in circumferential lumbar spinal fusion with minimum 2-year clinical results. Eur Spine J 14:727-737, 2005

28. Polsky D, Glick H: Costing and cost analysis in randomized controlled trials: caveat emptor. Pharmacoeconomics 27:179-188, 2009

29. Rabin R, de Charro F: EQ-5D: a measure of health status from the EuroQol Group. Ann Med 33:337-343, 2001

30. Reitman CA, Watters WC III, Sassard WR: The Cell Saver in adult lumbar fusion surgery: a cost-benefit outcomes study. Spine (Phila Pa 1976) 29:1580-1584, 2004

31. Resnick DK, Choudhri TF, Dailey AT, Groff MW, Khoo L,
Matz PG, et al: Guidelines for the performance of fusion procedures for degenerative disease of the lumbar spine. Part 3: assessment of economic outcome. J Neurosurg Spine 2:647652,2005

32. Russell LB, Gold MR, Siegel JE, Daniels N, Weinstein MC: The role of cost-effectiveness analysis in health and medicine. JAMA 276:1172-1177, 1996

33. Rutigliano MJ: Cost effectiveness analysis: a review. Neurosurgery 37:436-444, 1995

34. Savvidou C, Chatziioannou SN, Pilichou A, Pneumaticos SG: Efficacy and cost-effectiveness of cell saving blood autotransfusion in adult lumbar fusion. Transfus Med 19:202-206, 2009

35. Schulman KA: Medicare and cost-effectiveness analysis. $\mathbf{N}$ Engl J Med 354:207-209, 2006 (Letter)

36. Soegaard R, Bünger CE, Christiansen T, Høy K, Eiskjaer SP, Christensen FB: Circumferential fusion is dominant over posterolateral fusion in a long-term perspective: cost-utility evaluation of a randomized controlled trial in severe, chronic low back pain. Spine (Phila Pa 1976) 32:2405-2414, 2007

37. Suhonen R, Virtanen H, Heikkinen K, Johansson K, Kaljonen A, Leppänen T, et al: Health-related quality of life of day-case surgery patients: a pre/posttest survey using the EuroQoL-5D. Qual Life Res 17:169-177, 2008

38. Testa MA, Simonson DC: Assesment of quality-of-life outcomes. N Engl J Med 334:835-840, 1996

39. Tosteson AN, Lurie JD, Tosteson TD, Skinner JS, Herkowitz $\mathrm{H}$, Albert T, et al: Surgical treatment of spinal stenosis with and without degenerative spondylolisthesis: cost-effectiveness after 2 years. Ann Intern Med 149:845-853, 2008

40. Tosteson AN, Skinner JS, Tosteson TD, Lurie JD, Andersson $\mathrm{GB}$, Berven $\mathrm{S}$, et al: The cost effectiveness of surgical versus nonoperative treatment for lumbar disc herniation over two years: evidence from the Spine Patient Outcomes Research Trial (SPORT). Spine (Phila Pa 1976) 33:2108-2115, 2008

41. Tumeh JW, Moore SG, Shapiro R, Flowers CR: Practical approach for using Medicare data to estimate costs for cost-effectiveness analysis. Expert Rev Pharmacoecon Outcomes Res 5:153-162, 2005

42. Ubel PA, Hirth RA, Chernew ME, Fendrick AM: What is the price of life and why doesn't it increase at the rate of inflation? Arch Intern Med 163:1637-1641, 2003

43. Wang MY, Cummock MD, Yu Y, Trivedi RA: An analysis of the differences in the acute hospitalization charges following minimally invasive versus open posterior lumbar interbody fusion. Clinical article. J Neurosurg Spine 12:694-699, 2010

44. Wang MY, Lerner J, Lesko J, McGirt MJ: Acute hospital costs after minimally invasive versus open lumbar interbody fusion: data from a US national database with 6106 patients. J Spinal Disord Tech 25:324-328, 2012

45. Yao P, Wiggs BR, Gregor C, Sigurnjak R, Dodek P: Discordance between physicians and coders in assignment of diagnoses. Int J Qual Health Care 11:147-153, 1999

Manuscript submitted March 11, 2014.

Accepted April 1, 2014.

Please include this information when citing this paper: DOI: 10.3171/2014.4.SPINE14259.

Address correspondence to: Michael G. Kaiser, M.D., Columbia University, Neurological Surgery, The Neurological Institute, 710 W. 168th St., New York, NY 10032.email:mgk7@columbia.edu. 